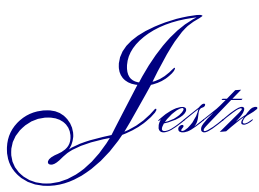

Journal of Engineering Science and Technology Review 1 (2008) 28-32

JOURNAL OF

Engineering Science and

Technology Review

Research Article

www.jestr.org

\title{
Superplasticity Effects And Strain Rate Dependency In A Material Joining Process
}

\author{
A. Vairis* \\ Mechanical Engineering Dept. TEI of Crete PO Box 1939, Heraklion 71004, Greece.
}

Received 10 January 2008; Accepted 20 February 2008

\begin{abstract}
The property of superplasticity of certain alloys, such as Ti6Al4V, where very large deformations can be reached before necking, has been used to manufacture complicated shapes in an economic fashion. In linear friction welding of Ti6A14V this property cannot be used to the full extent, as the strain rates encountered at the rubbing interface during joining affect the yield stress of the material in an adverse way, thus limiting the operating range of this type of welding equipment.
\end{abstract}

Keywords: superplasticity, Ti6Al4V, linear friction welding, strain rate dependent plastic deformation.

\section{Introduction}

It has been known since Joule's experiments, that mechanical energy can be partially transformed into heat generated from friction and much effort has gone into reducing friction by lubrication to prevent seizure. In most circumstances the thermal energy generated by rubbing contact of surfaces is regarded as undesirable, but it has been shown that it can be used to join materials by encouraging seizure in a controlled way which leads to efficient welding. Current welding methods fall into two classes, fusion welding or pressure welding. In pressure welding, joins are formed through mechanical plastic deformation in the weld zone brought about by the application of large forces. Contact surfaces are first brought into intimate contact by breaking down surface asperities and contaminants, so that subsequently the interatomic forces provide the bond. Friction provides the means to remove asperities by the mechanism of wear, and is used to generate heat to soften the materials to be joined before deforming them plastically. This dynamic mode of heat generation depends on a number of process parameters to be successful, as well as on the material properties of the parts to be joined at the temperature and stress conditions that the welding process is effecting on the worlpieces.

\section{Linear friction welding}

Friction welding is a solid state process for joining materials together, through intimate contact of a plasticised interface. The appropriate interface condition is generated by heat produced from frictional contact as one component is moved relative to, and in pressure contact with, the mating surface. The weld is usually consolidated by forging force, applied after the cessation of relative motion. The joint created, has a confined heat affected zone, and exhibits plastically deformed material around the weld which has been expelled due to the high internal pressures.

In linear friction welding parts move relative to each other under pressure in a direct reciprocating mode produced by a linear mechanism. It is a self regulating joining process for metals and plastics, where certain stress conditions at the interface and the region adjacent to it have to be reached for the process to undergo its various phases. The process is observed [1] to have four distinct phases (see Fig. 1):
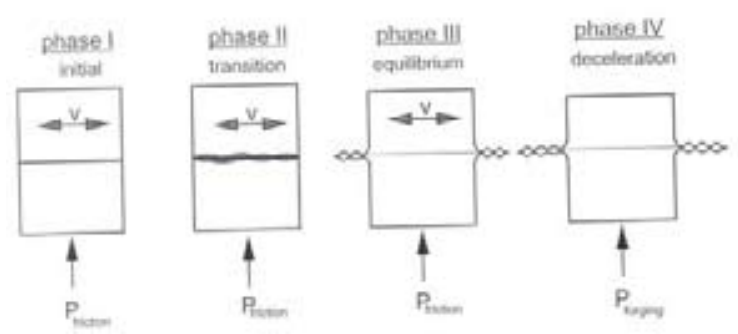

Fig. 1 Schematic depiction of the four distinct phases that are incorporated in the linear friction welding process. Specimens oscillate under pressure, which causes the temperature at the interface to increase due to friction and once it reaches an equilibrium it produces plastic material at the sides, which extrudes from the rubbing interface due to pressure and movement. The specimens are brought to rest quickly while applying forging pressure.

Phase I, The Initial Phase At the beginning the two materials are brought in contact under pressure. The two surfaces rest

\footnotetext{
*E-mail address: vairis@stef.teiher.gr

ISSN: 1791-2377 @ 2008 Kavala Institute of Technology. All rights reserved.
} 
on asperities, and heat is generated from solid friction. The true contact area is expected to increase throughout this phase. There is no axial shortening of the specimens at this stage. If the rubbing speed is too low for a given axial force, insufficient frictional heat will be generated to compensate for the conduction and radiation losses, which will lead to insufficient thermal softening and the next phase will not follow.

Phase II, The Transition Phase If sufficient frictional heat has been produced during the previous phase to soften the interface material, large wear particles begin to be expelled from the interface, and the heat affected zone expands for phase III to follow. The true contact area is considered to be $100 \%$ of the cross-sectional area, and the soft plasticised layer formed between the two materials at the interface is no longer able to support the axial load.

Phase III, The Equilibrium Phase Following phase II, axial shortening of the workpieces begins as a result of the expelled upset. In the plasticised layer formed at the interface, the local stress system with the assistance of the oscillatory movement extrudes material from the interface into the flash. Instabilities can appear at this stage, due to uneven distribution of temperature. If the temperature increases excessively in one part of the interface away from the centre line of oscillation, the plasticised layer becomes thicker in that section causing more plastic material to be extruded, which results in rotation of the interface from the original plane.

Phase IV, The Deceleration Phase Once the desired upset or predetermined process time is reached the oscillating workpiece is brought to rest very rapidly, and forging pressure may be applied to consolidate the weld.

There is a power input limit, below which welding is not possible. If operating below this limit either by using a smaller amplitude of oscillation, or rubbing at a lower frequency of oscillation, or applying a smaller friction pressure than necessary, the workpieces will never reach such conditions which will produce well defined flash and subsequently join to form sound welds.

The material studied in this work is Ti6Al4V (IMI 318), the most commonly used titanium alloy in the aerospace industry. Experiments were performed using a high frequency linear friction welding rig (see [1;2] for details of experiments) at $293 \mathrm{~K}$ (room temperature) and prevailing atmospheric conditions. The amplitude of oscillation used was $0.92 \mathrm{~mm}$ and $3 \mathrm{~mm}$, and the explored frequency range was from $10 \mathrm{~Hz}$ to $199 \mathrm{~Hz}$, well above commercially available linear friction welding machinery.

\section{Material extrusion of plastic material}

A successful weld is defined as one having permanent adhesion. All successful Ti6Al4V welds demonstrate of appreciable flash from both sides of the joint with the flash length being larger in the direction of movement. Extrusion is expected to occur in the middle point of oscillation, where the rubbing velocity is at its highest value and therefore the heat produced maximum. This increases the temperature instantaneously causing more material to yield. The flash does not have a uniform thickness but appears in the form of ridges. Extruded matter from the non-moving sides of the specimen which are parallel to the direction of movement also show some evidence of ridges. As there is no movement of the specimens in that direction, it may be concluded that axial shortening proceeds in a step-wise fashion. The effect of this pumping action is that material yields and extrudes in pulses, therefore confining the heat affected zone close to the interface.

The heat affected zone of the rubbing interface can be divided into the following sections:

- The plastic section at the interface. The strain rate is influenced by the rubbing velocity $v$, the axial shortening velocity $v_{0}$ and the rate that the material is expelled from the stationary side of the specimens. Material extrusion into the flash is due to the existence of this section.

- The partially deformed section next to the plastic section at the interface. The plastic deformation is limited by the axial shortening velocity $v_{0}$ and the rate that the material is expelled from the stationary side of the specimens.

The main components of strain rate have been calculated with an analytic model [3] for two experiments done at 0.92 $\mathrm{mm}$ amplitude of oscillation and frequencies of $65 \mathrm{~Hz}$ and $100 \mathrm{~Hz}$ (see Fig. 2). The effect of the higher frequency of oscillation is shown in the predicted strain rates in both directions. When the frequency of oscillation is $100 \mathrm{~Hz}$ both strain rate components were below $3.5 \mathrm{~s}^{-1}$. Using this analytical model it is indicated that higher strain rates are encountered at the interface with increasing frequency of oscillation.

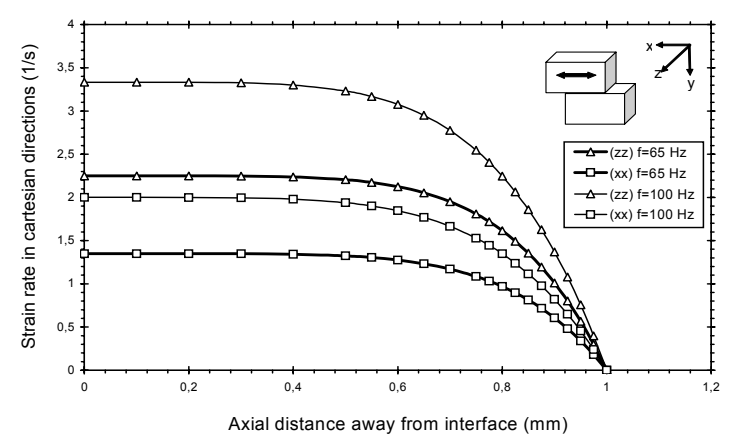

Fig. 2 Prediction of strain rate that the material is experiencing, using the analytical model, at various distances away from the rubbing interface for two directions ( $\mathrm{zz}$ and $\mathrm{xx}$ ) and two oscillating frequencies of linear friction welding $(1 / \mathrm{s})$

\section{Superplasticity}

Superplasticity is the phenomenon where crystalline solids deform in tension to very large plastic strains, often well in excess of $1000 \%$ (approaching $8000 \%$ in some alloys) under certain conditions of strain rate and temperature. Such a state is usually achieved at high homologous temperature, typically half the absolute melting point. This deformation results from the ability of the material to resist localized deformation much the same way that hot glass does. As large deformations are possible, complex contoured parts can be formed in a single forming cycle often eliminating the need for multipart fabrications.

Examples of superplastic materials are some fine-grained metals and ceramics. Other non-crystalline materials (amorphous) such as silica glass ("molten glass") and polymers also deform similarly, but are not called superplastic, because they are not crystalline; rather, their deformation is often described as Newtonian flow. Superplastically de- 
formed material gets thinner in a very uniform manner, rather than forming a 'neck' (a local narrowing) which leads to fracture. Also, the formation of internal cavities, which is another cause of early fracture, is inhibited.

In metals and ceramics, requirements for them being superplastic include a fine grain size $(\sim<20 \mu \mathrm{m})$ and a fine dispersion of thermally stable particles which act to pin the grain boundaries and maintain the fine grain structure at the high temperatures required for superplastic deformation. Those materials which meet these parameters must still have a strain rate sensitivity (a measurement of the way the stress on a material reacts to changes in strain rate) of $>0.3$ to be considered superplastic.

Initially superplastic deformation was considered as unique phenomenon inherent only to several alloys. However, systematic studies displayed its more general character as compared to conventional deformation. In reality, this effect can be observed not only in metals but in intermetallides and ceramics as well $[4,5]$ which, as known, are characterized by brittle failure under common conditions and display no features of plastic flow.

The physical nature of this phenomenon is very complex. Just recently $[6,7,8]$ it seemed that superplastic deformation could be explained by the operation of usual mechanisms of deformation, namely, grain boundary sliding (GBS), intragranular dislocation slip and diffusion creep. However, the latest data have shown that this phenomenon is conditioned by the operation of a specific mechanism of deformation cooperative grain boundary sliding (CGBS) [9]. The operation of this deformation mechanism does not depend on the crystal lattice type and dislocations present. It depends on the long-range area and structure of grain boundaries.

Superplasticity can be used by the design engineer to incorporate several detail parts into one piece structure. Thus materials with superplastic properties can be used to form complex components in shapes that are very near the final dimensions. Superplastic forming also enhances design freedom, minimizing the amount of scrap produced, and reducing the need for machining.

\section{Material Properties Of Ti6Al4V}

Ti6Al4V is an $\alpha+\beta$ alloy with good elevated temperature properties. In a manner similar to that in most metallic materials, the strength of $\mathrm{Ti} 6 \mathrm{Al} 4 \mathrm{~V}$ tends to decrease with increasing temperature. At temperatures in and above the agehardening range, time becomes an influential variable. As the times involved in the linear friction welding are less than one-half hour [10], only short time effects are concerned. Its ductility and toughness are influenced by the temperature that it has been exposed, due to phase transformations occurring above the beta transus temperature. The material has been shown to be strain rate sensitive at high strain rates, with no strain history effects on this sensitivity [11].

Temperatures encountered in friction welding of Ti6Al4V are well into the superplastic range, with the material exhibiting this behaviour. During superplasticity, necking is delayed during plastic deformation. In areas where necking has begun already, the local reduction in the cross sectional area causes an increase in the local strain rate. Therefore, an increase in stress is required to continue deforming at the reduced cross sectional area. The higher the strain rate sensitivity value, the higher is the local stress which would be required for neck formation. The stress which is required to maintain deformation is mainly depend- ent on the level of the deformation rate and is independent of the instantaneous strain. Strain rate sensitivity values are derived from the flow stress and the corresponding strain rate

$$
m=\frac{d \ln \sigma_{f}}{d \ln \dot{\varepsilon}}
$$

where $\sigma_{\mathrm{f}}$ is the flow stress.

Characterisation of stress / strain rate behaviour can be made with step strain rate tests, where the strain rate is increased in consecutive steps and the steady (or saturated) flow stress is recorded. Unfortunately, with these kinds of tests strain can become a variable along the stress - strain rate curve. Even when load relaxation tests results are used to derive the curves, more complex transient effects might be associated with the load relaxation tests [12] producing erroneous results for applications where strain rates increase with accompanying strain.

In [13] was the first published data on the superplastic behaviour of titanium and zirconium alloys. From a large number of tests at $800-1000^{\circ} \mathrm{C}$, they observed a variation in the values of the strain rate sensitivity as a function of temperature. They related the rapid decrease in the strain rate sensitivity value to the transformation from alpha to beta phase. Experimental values of flow stresses were close to those reported by [14] for the same temperatures and strain rates. As temperature increases above beta transus, strain rate sensitivity decreases. In the beta region the structure coarsens, which is associated with low strain rates, while more refined structures were developed at higher strain rates. A series of compression tests were performed on Ti6Al4V ELI [14] for temperatures between 800 and $1080^{\circ} \mathrm{C}$ and strain rates between 0.001 and $25 \mathrm{~s}^{-1}$, which are close to those encountered in linear friction welding. From the true stress - true strain flow curves, the strain rate sensitivity of the material is clearly exhibited (see Fig. 3 and Fig. 4). At low strain rates $\left(0.001,0.01,0.1\right.$ and $\left.1 \mathrm{~s}^{-1}\right)$ and for the isothermal compressions at 800,850 and $900^{\circ} \mathrm{C}$ the material strain softens after yielding. As the temperature of the experiment increases to $950,1000,1040$ and $1080^{\circ} \mathrm{C}$ behaviour changes to strain hardening after yielding for the same strain rates. At the high strain rate of $25 \mathrm{~s}^{-1}$ the material has a small strain hardening region, followed by a plateau and softening after the small peak flow stress, for all temperatures. The strain rate of $5 \mathrm{~s}^{-1}$ follows an intermediate behaviour. At temperatures of 800 and $850^{\circ} \mathrm{C}$ has a true stress true strain curve similar to the high strain rate tests, but from $900^{\circ} \mathrm{C}$ and onwards strain hardens as the lower strain rate tests.

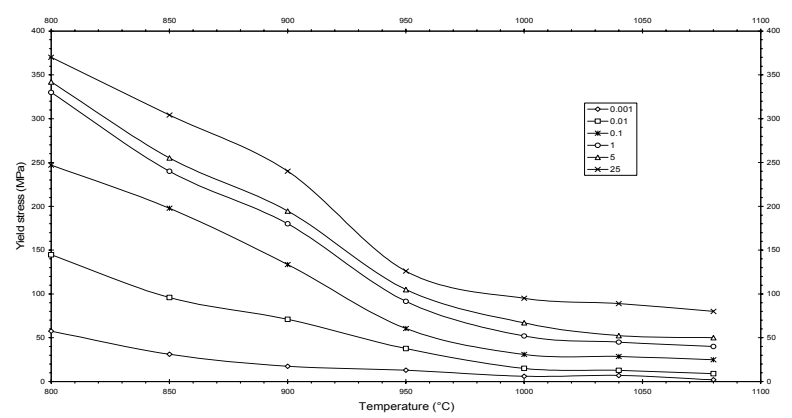

Fig. 3 Change of yield stress of Ti6Al4V for various temperatures at constant strain rates [14] 


\section{Results and Discussion}

Linear friction welding is a self regulating process, where certain stress conditions at the interface and the region adjacent to it have to be reached for the process to undergo its various phases. There is a power input limit, below which welding is not possible. If operating below this limit either by using smaller amplitude of oscillation, or rubbing at a lower frequency of oscillation or applying a smaller friction pressure than necessary, the specimens will never reach such conditions which will produce well defined flash and subsequently join to form sound welds. Local hot spots appear at the interface during phase I, from where hot material is extruded. These plasticised areas never extend to cover the whole of the rubbing interface. The result is to extrude material from all sides of the specimen, originating in these areas only.

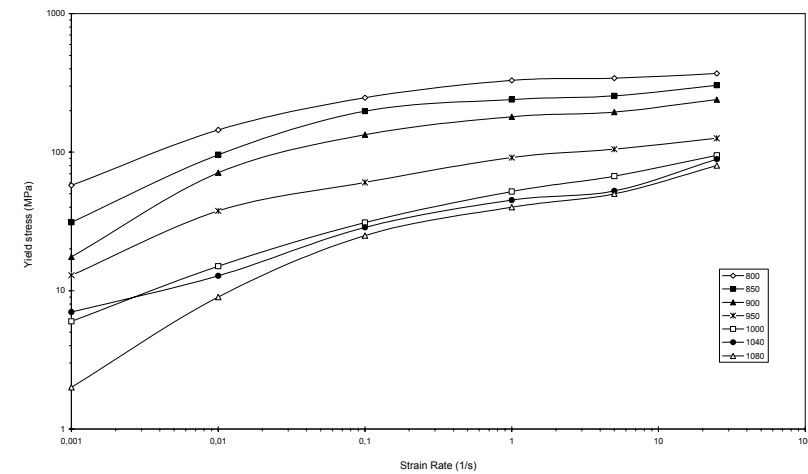

Fig. 4 Change of yield stress of Ti6Al4V for different strain rates at constant temperatures[14]

It was shown experimentally [1] that a critical heat flux is required to successfully weld superplastic Ti6Al4V. This heat flux is proportional to the axial load, as well as to the amplitude and frequency of oscillation. It follows that an increase in frequency might allow the axial forces to be reduced, making it possible to weld relatively delicate components like turbine blades, successfully without buckling. If the amplitude of oscillation were to be reduced, the frequency would necessarily need to increase proportionally for the same axial load, leading to similar conclusions. For the tests performed with Ti6A14V with oscillating frequencies up to $119 \mathrm{~Hz}$, the minimum power input to reach welding conditions and achieve a sound joint was found to increase with frequency.

This effect can be attributed to the strain rate sensitivity of the material, which works in an opposite way to the superplastic behaviour of the alloy. The strain rate sensitivity of this alloy has been documented at high temperatures and at strain rates between 0.001 and $25 \mathrm{~s}^{-1}$, where the compressive yield stress increases from $10 \mathrm{MPa}$ during static loading to $255 \mathrm{MPa}$ at $850^{\circ} \mathrm{C}$ for a strain rate of $5 \mathrm{~s}^{-1}$. The maximum theoretical strain rate that the hot material could experience in linear friction welding would be of that order.

As the material heats up to high temperatures due to friction, the yield stress of the material at the interface drops below the effective stress at the rubbing interface. This results in plastic deformation and subsequently this material is extruded under the combined action of hydrostatic pressure and oscillatory movement. The effect of the strain rate sensitive behaviour will be that higher friction pressures will need to be applied for yielding to occur at the same interface temperature as before. Alternatively, higher interface tempera- tures will have to be attained to compensate for the increase in yield stress.

As the two specimens oscillate in a sinusoidal manner, the maximum strain encountered at the rubbing interface, assuming no slippage, will be during the extreme point of oscillation where the distance from the middle point will equal the amplitude of oscillation. The maximum deformation that the material would be exposed to will equal the distance traveled from the opposite extreme point of oscillation, and will be two amplitudes of oscillation.

As $\mathrm{Ti} 6 \mathrm{Al} 4 \mathrm{~V}$ is strain rate sensitive, the power input to reach welding conditions and produce joints increases with frequency of oscillation for given amplitude in linear friction welding. The increased power requirements observed in the experiments indicate a linear relationship with frequency. If delicate components are to be welded, for which smaller friction pressures may be necessary, the frequency of oscillation should be increased for power input to remain constant. As the strain rate sensitivity of the titanium alloy would actually require increasing the friction pressure, this could be avoided by using longer contact lengths. The effect would be to compensate for the increase in strain rates due to frequency, and keep the strain rates constant.

\section{Conclusions}

Based on the linear friction welding experiments done with Ti6Al4V a number of points were concluded:

- The power input required to produce sound welds increases with increasing frequency of oscillation for the same amplitude of oscillation and friction pressure.

- Strain rates were predicted using experimental data, which verified the effect the frequency of oscillation has on strain rates that the plastic material at the interface is exposed to

- Superplasticity improves the manufacturing ability of certain alloys but may be limited by the strain rate dependency of yield stress at the process temperature

Acknowledgments: The author would like to thank the Commission of the European Communities for the financial support provided in the form of a Research Fellowship within the Human Capital Mobility Programme and the University of Bristol for providing laboratory facilities. 


\section{References}

1. A. Vairis, M. Frost, Wear, 217, 117 (1998).

2. A.Vairis, M.Frost, Journal of Materials and Manufacturing Processes, 21, 766 (2006).

3. A.Vairis, M.Frost, Materials Science and Engineering: A, 292, 8 (2000).

4. O.A. Kaibyshev, Superplasticity of Alloys, Intermetallides, and Ceramics, Springer-Verlag, Berlin/New York, 317 (1992)

5. A.K. Mukherjee, in: H. Mughrabi (Ed.), Plastic Deformation and Fracture of Materials, vol. 6, Materials Science and Technology, VCH Verlagsgesellschafi mbH, Germany, (1993)/

6. I.I. Novikov, V.K. Portnoy, Superplasticity of Alloys with Ultrafined Grains, Metallurgia, Moscow, 168 (1981).

7. T.G. Langdon, Key Eng. Mater. 109 (1994)

8. A. Kaibyshev, Superplasticity of Commercial Alloys, Metallurgia, Moscow, 264 (1984).
9. V.V. Astanin, O.A. Kaibyshev, S.N. Faizova, Scripta Metall. Mater. 25, 2663 (1991).

10. D.J., Maykuth, "Ti-base alloys : Ti 6Al 4V Processes and properties handbook", DMIC Publication , (1971).

11. J. Duffy, Mechanical Properties At High Rates Of Strain, Institute of Physics Conference Series No.47, 1 (1979).

12. A.K. Ghosh,. C.H. Hamilton, Metallurgical Transactions A, 13A, 733 (1982).

13. D. Lee, W.A. Backofen, Transactions Of The Metallurgical Society Of AIME, 239, 1034 (1967).

14. P. Chaudhury, D. Zhao, "Atlas of formability : Ti 6Al 4V ELI", National Center for Excellence in Metalworking Technology (1992) 